\title{
A robust asymptotic observer for systems that converge to unobservable states. A batch reactor case study
}

\author{
ALAIN RAPAPORT and DENIS DOCHAIN
}

\begin{abstract}
In this paper we propose an observer for a dynamical system for which the states on the frontier of its domain are not observable, and all trajectories converge to the frontier. The proposed case study, a bioreactor in batch operating conditions with a single microbial reaction and gas production, is standard and largely encountered in practical situations. We show also how to extend this observer to obtain an observer in higher dimension that is robust with respect to unbiased noise.
\end{abstract}

Index Terms-State observer, observability, biological systems.

\section{INTRODUCTION}

The state observation of nonlinear systems is a wide and active research area resulting in a large number of scientific publications on the subject, e.g. [9] [11], [13] to mention a few, as well as the interesting and nicely written survey paper by Krener [14]. Observers are often used in application fields for which sensors are missing, such as typically in chemical and biochemical engineering (see for instance [2], [5] and the recent survey paper [1]). In particular, observers have been proposed for continuously fed bioreactors (see for instance the application of the "high-gain observer" in [8]), but comparatively less work has considered batch reactors (even though it may require to setup some unstraightforward extensions of the theory [18]). Let us also underline that almost all theoretical and applied contributions require the system to be observable. In the present paper, we address a state observation issue that, to the best of our knowledge, has not yet been addressed, i.e. the problem of the on-line reconstruction of the variables of a nonlinear system for which there is a loss of observability on the frontier of its domain, which, moreover, is attracting. We shall indeed concentrate on a biological model with one biomass $x$, one substrate $s$ and gaseous outflow rate $y$ (measured on-line), and for which the initial condition $\left(x_{0}, s_{0}\right)$ is unknown. Incidentally the design of the state estimate takes advantage of the fundamental reaction invariant property of reaction systems [10]. The conference paper [7] presents a new approach for the design of an asymptotic observer in this context of lack of observability, but that is unfortunately not robust to measurement errors. The present paper develops a robust extension of this first approach.

A. Rapaport (corresponding author) is with MISTEA, Univ Montpellier, INRA, Montpellier SupAgro, France. E-mail: alain.rapaporteinra.fr

D; Dochain is with ICTEAM, Université catholique de Louvain, Belgium. E-mail: denis.dochain@uclouvain.be
The paper is organized as follows. Section II introduces the dynamical model of the biological system. Section III provides an analysis of its observability properties. An asymptotic observer is derived in Section IV. Then a robust version of this observer, in higher dimension, is derived in Section V. Computational aspects for the implementation of the observer are considered in Section VI. Section VII studies Luenberger observers as alternatives of the proposed observer. Finally Section VIII provides several numerical simulations to illustrate the performance of the proposed observer as well as its comparison with the Luenberger observers designed in Section VII.

\section{DYNAMICAL MODEL}

Let us consider the dynamical model of a simple microbial growth reaction in a batch reactor (see e.g. [2]):

$$
\left\{\begin{array}{l}
\dot{x}=\mu(s) x \\
\dot{s}=-\mu(s) x
\end{array}\right.
$$

where $x$ and $s$ stand for the concentrations in biomass and substrate, respectively. Without loss of generality, we assume that the yield coefficient of the transformation of the substrate into biomass is equal to 1 . The specific growth rate function $\mu(\cdot)$ satisfies the usual assumption:

Assumption 2.1: The function $\mu(\cdot)$ is Lipschitz continuous on $\mathbb{R}_{+}$, positive on $(0,+\infty)$ with $\mu(0)=0$.

Although state estimation for bioprocesses has received a great interest in the literature (see e.g. [22], [6]), most of the contributions concern continuous reactors. There are comparatively much less works for batch processes, apart from [12], [21], [17], [4].

In the present work, we consider that a gaseous by-product flow rate, such as biogas (e.g. [3]), is measured on-line as a quantity proportional to the output variable

$$
y=\mu(s) x .
$$

Typically, it often happens in batch bioprocesses that the initial quantities of reactants $\left(x_{0}, s_{0}\right)$ are unknown and that the biogas production is the only available measurement during the course of the fermentation. However, such kind of measurement is not considered in the references previously cited. As the solutions of (1) clearly satisfy $\lim _{s \rightarrow+\infty} s(t)=0$, the initial quantity of substrate can be recovered as

$$
s_{0}=\lim _{t \rightarrow+\infty} \int_{0}^{t} y(\tau) d \tau
$$


However the main interest is usually to estimate the total production of biomass, that is $\lim _{t \rightarrow+\infty} x(t)$. The purpose of the present work is the present a simple and reliable methodology to estimate the biomass concentration $x$.

\section{OBSERVABILITY ANALYSIS}

Equivalently one can consider the dynamics in the $(z, s)$ coordinates with $z=x+s$

$$
\begin{aligned}
& \frac{d}{d t}\left[\begin{array}{l}
z \\
s
\end{array}\right]=f(z, s):=\left[\begin{array}{c}
0 \\
-\mu(s)(z-s)
\end{array}\right], \\
& y=h(z, s):=\mu(s)(z-s),
\end{aligned}
$$

on the positive cone

$$
\mathcal{C}:=\left\{(z, s) \in \mathbb{R}^{2} \mid z>s>0\right\} .
$$

Here we require a stronger assumption on the function $\mu$.

Assumption 3.1: The function $\mu$ is $C^{2}$, concave and increasing on $\mathbb{R}_{+}$, with $\mu(0)=0$.

For convenience, we denote the number

$$
\bar{\mu}=\max _{s>0} \mu(s)
$$

(that could be finite or not). Such an assumption is for instance fulfilled for the well-known Monod function

$$
\mu(s)=\frac{\bar{\mu} s}{K_{s}+s}
$$

but not for Haldane or Hill functions (see Section VIII).

Let us recall the usual definition of differential observability (see [9]). The pair $(f, h)$ is differentially observable on a domain $\mathcal{D}$ when it exists an integer $n$ such that the map $x \mapsto\left(h(x), L_{f}(x), \cdots f_{f}^{n} h(x)\right)$ is injective on $\mathcal{D}$, where $L_{f} h$ denotes the Lie derivative of $h$ with respect to $f$ : $L_{f} h(x)=\partial_{x} h(x) . f(x)$ and $L_{f}^{i} h(x)=L_{f}\left(L_{f}^{i-1} h\right)(x)$.

Lemma 3.1: Under Assumption 3.1, the system (2)(3) is differentially observable on $\mathcal{C}$, but not on $\partial C$. can write

Proof: On the open cone $\mathcal{C}$, one has $\mu(s)>0$ and we

$$
\begin{aligned}
L_{f} h(z, s) & =-\left[\mu^{\prime}(s)(z-s)-\mu(s)\right] \mu(s)(z-s) \\
& =-\left(\frac{\mu^{\prime}(s)}{\mu(s)} h(z, s)-\mu(s)\right) h(z, s) .
\end{aligned}
$$

For a given non-negative number $y$, we define the function

$$
\varphi_{y}(s):=\mu(s)-\frac{\mu^{\prime}(s)}{\mu(s)} y
$$

whose derivative is

$$
\varphi_{y}^{\prime}(s)=\mu^{\prime}(s)-\left(\frac{\mu^{\prime \prime}(s)}{\mu(s)}-\frac{\mu^{\prime}(s)^{2}}{\mu(s)^{2}}\right) y .
$$

Under Assumption 3.1, $\varphi_{y}^{\prime}(s)$ is positive for any $s$, and therefore the inverse $\varphi_{y}^{-1}$ is well defined from $\varphi\left(\mathbb{R}_{+}\right)$to $\mathbb{R}_{+}$. As $h(z, s)>0$ on $\mathcal{C}$, one can write

$$
\left\{\begin{array}{l}
s=\varphi_{h(z, s)}^{-1}\left(\frac{L_{f} h(z, s)}{h(z, s)}\right), \\
z=\frac{h(z, s}{\mu(s)}+s,
\end{array}\right.
$$

and conclude that the map

$$
\left[\begin{array}{l}
z \\
s
\end{array}\right] \mapsto\left[\begin{array}{c}
h(z, s) \\
L_{f} h(z, s)
\end{array}\right]
$$

is injective on $\mathcal{C}$, and that is the system is differentially observable on $\mathcal{C}$.

On the frontier of $\mathcal{C}$, one has $L_{f^{i}} h \equiv 0$ for any integer $i$ and therefore the system is not differentially observable on $\partial \mathcal{C}$.

One can note that the boundary $z=s$ (that is $x=0$ ) of the cone $\mathcal{C}$ is invariant but repulsive for the dynamics, while the boundary $s=0$ is attractive. Therefore for any initial condition with $z_{0}>s_{0}$ (that is $x_{0}>0$ ), the solution converges to the boundary $s=0$ where the system is no longer observable. This feature prevents classical derivations of nonlinear observers, such as the high-gain observer, that requires the (differential) observability on a compact invariant set (which here has to contain $s=0$ ). In the coming section, we design an observer with a completely different structure. Later on, in Section VII, we also consider two Luenberger observers as "naive" alternatives and show that one cannot always guarantee their convergence. Due to the lack of observability, one cannot expect to find an observer whose speed of convergence can be arbitrarily assigned. However we aim at proposing a robust estimator whose error converges asymptotically to zero, guaranteeing a small error when batch reactors are operated on sufficiently large time horizon.

\section{AN ASYMPTOTIC OBSERVER}

In this section, we first show that the system is detectable from any positive initial condition, in the sense of Proposition 4.1 given below, without requiring Assumption 3.1. Let us underline that the system is not detectable in the usual sense (see e.g. [23]). It is not neither weakly detectable i.e. detectable for initial conditions close from the steady state. Indeed the state vectors in $\mathbb{R}_{+} \times\{0\}$ are all indistinguishable steady states and trajectories of the system converges to one of them. This prevents the use of estimation techniques based on passivity that requires only detectability (see e.g. [16], [19], [20]).

Proposition 4.1: Under Assumption 2.1, when $y(0)>0$, one has

$$
\lim _{t \rightarrow+\infty} s(t)=0, \quad \lim _{t \rightarrow+\infty} x(t)=z,
$$

where

$$
z=\frac{y\left(t_{0}\right)}{\mu\left(\int_{t_{0}}^{+\infty} y(\tau) d \tau\right)}+\int_{t_{0}}^{+\infty} y(\tau) d \tau
$$

for any $t_{0}>0$.

Proof: When $y(0)>0$, one has $x_{0}>0$ and $s_{0}>0$. Clearly, the solution of (1) fulfills $(x(t), s(t)) \rightarrow(z, 0)$ when $t$ tends to $+\infty$, where $z=x_{0}+s_{0}$. Therefore we can write

$$
s\left(t_{0}\right)=\int_{t_{0}}^{+\infty} y(\tau) d \tau
$$

for any $t_{0}>0$, and then we also have

$$
x\left(t_{0}\right)=\frac{y\left(t_{0}\right)}{\mu\left(\int_{t_{0}}^{+\infty} y(\tau) d \tau\right)} .
$$


Finally we obtain the following exact expression for $z$ :

$$
z=x\left(t_{0}\right)+s\left(t_{0}\right)=\frac{y\left(t_{0}\right)}{\mu\left(\int_{t_{0}}^{+\infty} y(\tau) d \tau\right)}+\int_{t_{0}}^{+\infty} y(\tau) d \tau
$$

for any $t_{0}>0$, which completes the proof.

One can then consider the following asymptotic observer.

Proposition 4.2: Let Assumption 2.1 be fulfilled. For any initial condition $\left(x_{0}, s_{0}\right)$ with $x_{0}>0$ and $s_{0}>0$, the following observer

$$
\left\{\begin{array}{l}
\dot{v}(t)=y(t), \quad v(0)=0 \\
\hat{x}(t)=\frac{y(0)}{\mu(v(t))}+v(t) \quad(t>0)
\end{array}\right.
$$

satisfies

$$
\lim _{t \rightarrow+\infty} \hat{x}(t)-x(t)=0 .
$$

If, in addition, the function $\mu$ is increasing, then the error $\hat{x}-x$ is decreasing with time.

Remark 4.1: The internal variable $v(\cdot)$ of the observer (7) is well defined for any $t \geq 0$, while the estimation $\hat{x}(\cdot)$ as "output" of this system is defined only for $t>0$, which is quite unusual in observer design. More precisely, one has $\lim _{t \rightarrow 0^{-}} \hat{x}(t)=+\infty$, but in practice the estimation $\hat{x}(\cdot)$ drops down very quickly from large values at small times $t>0$, as it can be seen in numerical simulations in Section VIII. This is not related to a "peaking phenomenon" in the dynamics (there is no "high-gain"), but is simply due to the fact that $\hat{x}$ is not defined at $t=0$.

Proof of Proposition 4.2: Note that for $t>0$, the solution of (7) is given by the expression

$$
\hat{x}(t)=\frac{y(0)}{\mu\left(\int_{0}^{t} y(\tau) d \tau\right)}+\int_{0}^{t} y(\tau) d \tau .
$$

The convergence of the observer is then a simple consequence of Proposition 4.1. The time derivative of the $x$-error is determined straightforwardly as

$$
\frac{d}{d t}(\hat{x}-x)(t)=-y(0) \frac{\mu^{\prime}\left(\int_{0}^{t} y(\tau) d \tau\right)}{\left[\mu\left(\int_{0}^{t} y(\tau) d \tau\right)\right]^{2}} y(t)
$$

which is negative when the function $\mu$ is increasing.

Remark 4.2: The fact that the error $\hat{x}-x$ is decreasing with time guarantees that $\hat{x}(t)$ is an upper estimation of $x(t)$ at any time $t$ and that the estimator does not oscillate as it could happen with high-gain observers. However its convergence speed cannot be tuned as for standard observers.

Remark 4.3: When the growth function $\mu$ depends on another substrate or resource $r$ that is assumed to be measured, and when the conditions for which $s$ tends asymptotically to 0 are fulfilled, then the estimator (7) can be straightforwardly extended:

$$
\left\{\begin{array}{l}
\dot{v}(t)=y(t), \quad v(0)=0 \\
\hat{x}(t)=\frac{y(0)}{\mu(v(t), r(t))}+v(t) \quad(t>0) .
\end{array}\right.
$$

The main drawback of the proposed observer is its sensitivity on the error on the initial measurement $y_{0}$ : unlike classical observers, the initial measurement $y\left(t_{0}\right)$ is not forgotten. Simulations performed in Section VIII show that when the initial error on $y_{0}$ is too large, its convergence is no longer guaranteed. We present now an extended version of the observer to cope with this drawback.

\section{A ROBUST ASYMPTOTIC OBSERVER}

We consider that the measurement is now given by

$$
y_{\text {obs }}(t)=y(t)+p(t)
$$

where $p(\cdot)$ is a disturbance. Even when the disturbance $p(\cdot)$ is unbiased, one can easily check that the observer $\hat{z}(\cdot)$ given in (7) with $y(\cdot)$ replaced by $y_{o b s}(\cdot)$ has an asymptotic bias:

$$
\lim _{t \rightarrow+\infty} \hat{x}(t)-x=\frac{p(0)}{\mu\left(\int_{0}^{+\infty} y(\tau) d \tau\right)} .
$$

We consider the following stronger assumption on the bias of the disturbance $p(\cdot)$, which is discussed later in Remark 6.1 .

Assumption 5.1: There exists $T<+\infty$ such that

$$
\frac{1}{T} \int_{t}^{t+T} p(\tau) d \tau=0, \quad \forall t>0
$$

We show that under this assumption, it is possible to derive an estimator without asymptotic bias.

Proposition 5.1: Let Assumption 2.1 be fulfilled. For any $p(\cdot)$ that satisfies Assumption 5.1, the following estimator

$\hat{x}(t)=\frac{\int_{t_{0}=0}^{t_{0}=T}\left\{y_{o b s}\left(t_{0}\right)+\int_{t_{0}}^{t} y_{o b s}(\tau) d \tau \cdot \mu\left(\int_{t_{0}}^{t} y_{o b s}(\tau) d \tau\right)\right\} d t_{0}}{\int_{t_{0}=0}^{t_{0}=T} \mu\left(\int_{t_{0}}^{t} y_{o b s}(\tau) d \tau\right) d t_{0}}$

which is defined for $t>T$, is unbiased.

Proof: First note that, due to Assumption 5.1, one has

$$
\int_{t_{0}=0}^{t_{0}=T} y_{o b s}\left(t_{0}\right) d t_{0}=\int_{t_{0}=0}^{t_{0}=T} y\left(t_{0}\right) d t_{0}
$$

and

$$
\int_{t_{0}}^{+\infty} y_{o b s}(\tau) d \tau=\int_{t_{0}}^{+\infty} y(\tau) d \tau, \quad \forall t_{0} \in[0, T] .
$$

Moreover, from equation (6), one has

$$
\mu\left(\int_{t_{0}}^{+\infty} y(\tau) d \tau\right) z=y\left(t_{0}\right)+\int_{t_{0}}^{+\infty} y(\tau) d \tau \cdot \mu\left(\int_{t_{0}}^{+\infty} y(\tau) d \tau\right)
$$

for any $t_{0} \in[0, T]$. Integrating this last inequality between $t_{0}=0$ and $t_{0}=T$ yields

$$
\begin{aligned}
& \int_{t_{0}=0}^{t_{0}=T} \mu\left(\int_{t_{0}}^{+\infty} y(\tau) d \tau\right) d t_{0} \cdot z \\
& \quad=\int_{t_{0}=0}^{t_{0}=T} y\left(t_{0}\right) d t_{0}+\int_{t_{0}=0}^{t_{0}=T} \int_{t_{0}}^{+\infty} y(\tau) d \tau \cdot \mu\left(\int_{t_{0}}^{+\infty} y(\tau) d \tau\right) d t_{0}
\end{aligned}
$$


or equivalently

$z=\frac{\int_{t_{0}=0}^{t_{0}=T} y\left(t_{0}\right) d t_{0}+\int_{t_{0}=0}^{t_{0}=T} \int_{t_{0}}^{+\infty} y(\tau) d \tau \cdot \mu\left(\int_{t_{0}}^{+\infty} y(\tau) d \tau\right) d t_{0}}{\int_{t_{0}=0}^{t_{0}=T} \mu\left(\int_{t_{0}}^{+\infty} y(\tau) d \tau\right) d t_{0}}$

which proves that one has

$$
\lim _{t \rightarrow+\infty} \hat{x}(t)=z
$$

and we conclude by Proposition (4.1).

Remark 5.1: The observer (10) is not an averaging of the observer proposed in Proposition 4.2, which can be equivalently written in the integral form (8). It is neither the expression (8) evaluated on an average of the measurements.

\section{COMPUTATIONAL ASPECTS OF THE ROBUST OBSERVER}

The robust observer given in Proposition 5.1 requires an integration on $t_{0}$ and therefore cannot be written as a solution of a dynamical system in finite dimension. However the integrals

$$
I\left(t_{0}, t\right)=\int_{t_{0}}^{t} y_{o b s}(\tau) d \tau, \quad t>t_{0}
$$

can be determined for $N$ discrete values of $t_{0}=$ $0, h, 2 h, \cdots, T-h$ with $h=T / N$, by integrating the variables $v_{i}$ solutions of the system of $N$ differential equations

$$
\dot{v}_{i}=y_{o b s(t)} \mathbb{1}_{\{t>(i-1) h\}}(t), \quad v_{i}(0)=0 \quad(i=1 \cdots N)
$$

where $\mathbb{1}_{A}(\cdot)$ denotes the indicator function of a set $A$. Clearly, one has

$$
v_{i}(t)=I((i-1) h, t), \quad t>(i-h) h \quad(i=1 \cdots N) .
$$

Then the integrals of $\gamma\left(I\left(t_{0}, t\right)\right)$ from $t_{0}=0$ to $t_{0}=T$, where $\gamma(\cdot)$ is any smooth function, are approximated by

$$
\int_{t_{0}=0}^{t_{0}=T} \phi\left(I\left(t_{0}, t\right)\right) \simeq h \sum_{i=1}^{N} \gamma\left(v_{i}(t)\right), \quad t>T
$$

and we obtain the following approximation of the estimator (10) under the form of a finite dimensional observer:

$$
\left\{\begin{array}{l}
\dot{v}_{i}=y_{o b s(t)} \mathbb{1}_{\{t>(i-1) h\}}(t), \quad v_{i}(0)=0 \quad(i=1 \cdots N), \\
\hat{x}(t)=\frac{\frac{N}{T} v_{1}(T)+\sum_{i=1}^{N} v_{i}(t) \mu\left(v_{i}(t)\right)}{\sum_{i=1}^{N} \mu\left(v_{i}(t)\right)} t>T,
\end{array}\right.
$$

Remark 6.1: In practice, one can choose $T$ large enough compared to the inverse of the fundamental frequency and first harmonics of the Fourier decomposition of the noise. This observer is an extension of the former one which is based on the single initial measurement $y(0)$, providing a filtering of this information. A large value of $T$ ensures robustness but at the cost of needing to wait this longer time before having a first estimate. In any case, the choice of $T$ gives flexibility to practitioners in the design of the observer.

\section{CONSIDERATION OF LUENBERGER OBSERVERS}

In this section, we consider functions $\mu(\cdot)$ that satisfy Assumption 3.1, so that the differential observability is fulfilled during the transient. We study the behavior of Luenberger observers when time tends towards infinity.

As we aim at reconstructing the biomass concentration $x$, we write the system (1) in $(x, y)$ coordinates as follows

$$
\left\{\begin{array}{l}
\dot{x}=y \\
\dot{y}=\phi(x, y) y
\end{array}\right.
$$

with

$$
\phi(x, y)=-\mu^{\prime} \circ \mu^{-1}\left(\frac{y}{x}\right) x+\frac{y}{x} .
$$

Note that $\mu^{-1}$ is well defined on $[0, \bar{\mu}]$ under Assumption 3.1. While the map $\phi$ has a singularity at $x=0$, solutions of (1) satisfy $x(t) \geq x_{0}$ at any $t>0$ and therefore avoid this singularity for any initial condition with $x_{0}>0$. For the design of an observer, one has to extend this dynamics for values of $x$ that are non positive or such that $y / x$ is larger than $\bar{\mu}$. Let $\epsilon$ be a positive number, and consider the map

$$
\tilde{\phi}(x, y)=\phi(\max (x, \epsilon, y / \bar{\mu}), y)
$$

which coincides with $\phi$ along any solution of (12) with $x_{0} \geq \epsilon$. Moreover $\tilde{\phi}$ is Lipschitz continuous on $\mathbb{R} \times \mathbb{R}_{+}$. We can then consider a Luenberger observer [15] in the $(x, y)$ coordinates for the dynamics with $\phi$ replaced by $\tilde{\phi}$ :

$$
\left\{\begin{array}{l}
\dot{\hat{x}}=y(t)+G_{1}(\hat{y}-y(t)), \\
\dot{\hat{y}}=\tilde{\phi}(\hat{x}, y(t)) y(t)+G_{2}(\hat{y}-y(t)),
\end{array}\right.
$$

Let us write the error dynamics as a time-varying linear system:

$$
\frac{d}{d t}\left[\begin{array}{l}
e_{x} \\
e_{y}
\end{array}\right]=\underbrace{\left[\begin{array}{cc}
0 & G_{1} \\
\delta(t) y(t) & G_{2}
\end{array}\right]}_{A(t)}\left[\begin{array}{l}
e_{x} \\
e_{y}
\end{array}\right]
$$

where the function $\delta(\cdot)$ has to be defined as follows:

$$
\delta(t)=\mid \begin{array}{ll}
\frac{\tilde{\phi}(\hat{x}(t), y(t))-\phi(x(t), y(t))}{\hat{x}(t)-x(t)} & \text { if } \hat{x}(t) \neq x(t), \\
\partial_{x} \phi(x(t), y(t)) & \text { if } \hat{x}(t)=x(t) .
\end{array}
$$

Under Assumption 3.1, the derivative $\mu^{\prime}$ is bounded on $\mathbb{R}_{+}$ and then the map $x \mapsto \tilde{\phi}(x, y)$ has linear growth for any $y \in \mathbb{R}_{+}$. As $y(\cdot)$ is bounded, $\delta(\cdot)$ is thus bounded whatever is the solution $\hat{x}(\cdot)$. By Assumption 2.1, one has $\mu^{-1}(0)=0$ from which we deduce $\phi(x, 0)=-\mu^{\prime}(0) x$ and $\tilde{\phi}(x, 0)=$ $-\mu^{\prime}(0) \max (\epsilon, x)$ for any $x>0$. As one has $\lim _{t \rightarrow+\infty} y(t)=$ 0 , we obtain

$$
\lim _{t \rightarrow+\infty} A(t)=\left[\begin{array}{ll}
0 & G_{1} \\
0 & G_{2}
\end{array}\right]
$$

which is not a Hurwitz matrix. The asymptotic convergence of the error towards 0 is thus not guaranteed and the choice of the gains $G_{1}, G_{2}$ does not allow to assign the speed of convergence, as this will be shown in the numerical simulations in the next section. 
Consider instead the dynamics (12) in $(x, w)=(x, \log y)$ coordinates, and the Luenberger observer

$$
\left\{\begin{array}{l}
\dot{\hat{x}}=y(t)+G_{1}(\hat{w}-\log y(t)), \\
\dot{\hat{w}}=\tilde{\phi}(\hat{x}, y(t))+G_{2}(\hat{w}-\log y(t)),
\end{array}\right.
$$

The error equation is then given by the dynamics

$$
\frac{d}{d t}\left[\begin{array}{c}
e_{x} \\
e_{w}
\end{array}\right]=\underbrace{\left[\begin{array}{cc}
0 & G_{1} \\
\delta(t) & G_{2}
\end{array}\right]}_{A(t)}\left[\begin{array}{c}
e_{x} \\
e_{w}
\end{array}\right]
$$

and one obtains (for $\epsilon$ sufficiently small)

$$
\lim _{t \rightarrow+\infty} A(t)=\left[\begin{array}{cc}
0 & G_{1} \\
-\mu^{\prime}(0) & G_{2}
\end{array}\right]
$$

The error dynamics converges asymptotically towards an autonomous linear dynamics, which is Hurwitz for growth functions with $\mu^{\prime}(0)>0$ and a suitable choice of the gains $G_{1}$, $G_{2}$. This ensures the asymptotic convergence of the observer in the $(x, w)$ coordinates, but note that the internal state $w$ of the observer is unbounded. We shall see in the next section that there are some issues for the practical implementation of the observer (14).

\section{NUMERICAL SIMULATIONS}

\section{A. Behavior of the asymptotic observer}

1) Under Assumption 3.1: Let us consider the Monod function (4) with $\bar{\mu}=1$ and $K_{s}=1$ (see Fig. 1). We

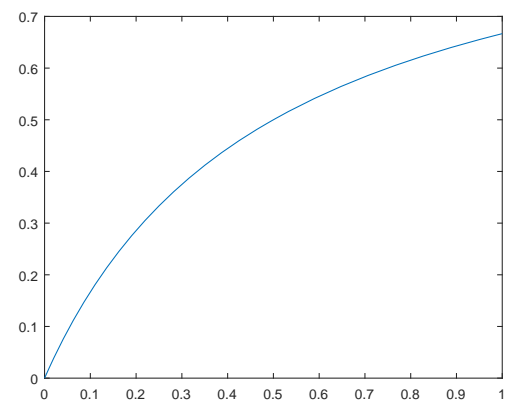

Figure 1. Graph of a Monod function

have considered the initial condition $\left(s_{0}, b_{0}\right)=(1.1,1.5)$ and compared the asymptotic observer (7) with the Luenberger observers (13) an (14) initialized with $(\hat{x}, \hat{y})=(1, y(0))$ for various gains $G_{1}, G_{2}$. Measurements have been randomly disturbed by a white noise proportional up to $10 \%$ of the signal. We found a systematic bias on the asymptotic error of the Luenberger observer (13), as depicted on Fig. 2 (for the empirical choice of $G_{1}=G_{2}=-20$ that gave the best result for this particular initial condition). These simulations show that the innovation $\hat{y}-y$ of the Luenberger observer (13) reaches zero while the $x$ error has not yet converged to zero, which illustrates its non null asymptotic error. For the Luenberger observer (14) in $(x, w)$ coordinates, one has $\mu^{\prime}(0)=\bar{\mu} / K_{s}>0$ which ensures its convergence for the choice of $G_{1}=\lambda_{1} \lambda_{2} / \mu^{\prime}(0)$ and $G_{2}=\lambda_{1}+\lambda_{2}$ with
$\operatorname{Re}\left(\lambda_{i}\right)<0$. However, we found its convergence much slower than the asymptotic observer if one does not choose very large values of $\lambda_{i}$ (at least -10 ). Unfortunately these large values make the estimation very sensitive to noise (see Fig. 2). Recall that the spectrum placement concerns the matrix $A(\infty)$ of the asymptotic error dynamics and does not guarantee that the transient speed can be arbitrarily assigned. Moreover, when the observation $y(t)$ takes the null value, the $\log y(t)$ term in (14) is not well defined. This condition would require modifications to the integration scheme.
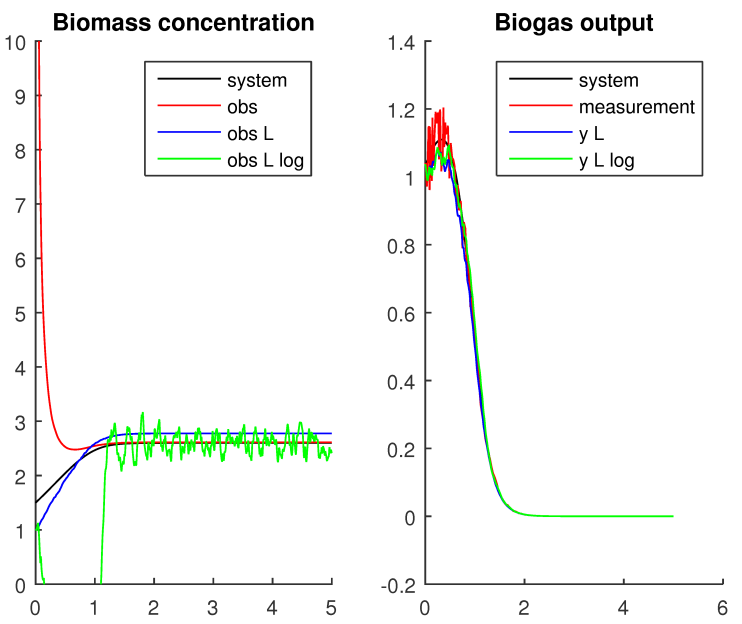

Figure 2. Comparison of the proposed observer with the Luenberger observers (13) and (14) denoted 'L' and 'L log' (with Monod function)

2) In absence of Assumption 3.1: We consider now two different growth functions $\mu(\cdot)$ that do not satisfy Assumption 3.1. Let us stress that this does not prevent the convergence of the asymptotic observer of Proposition 4.2, which requires Assumption 2.1 only to be fulfilled. The system remains detectable in the sense of Proposition 4.1, but the observability analysis of Section III can no longer be conducted. As a matter of comparison, we have also simulated the Luenberger observers.

The Hill function for the specific growth rate writes as follows (see Fig. 3): and is increasing but not concave (see Fig. 3).

$$
\mu(s)=\frac{\bar{\mu} s^{\alpha}}{K_{s}^{\alpha}+s^{\alpha}} \quad(\alpha>1)
$$

Note that one has $\mu^{\prime}(0)=0$. Therefore the convergence of the observer (14) in $(w, \log y)$ coordinates is not guaranteed. Simulations of Fig. 4 have been ran for $\bar{\mu}=1, K_{s}=0.5$ and $\alpha=2$.

The Haldane model for the specific growth rate (see Fig. 5) is given by the following expression:

$$
\mu(s)=\frac{\mu_{0} s}{K_{s}+s+\frac{s^{2}}{K_{i}}}
$$

and is non monotonic (see Fig. 5). Therefore the inverse of $\mu$ is not uniquely defined for the construction of the 


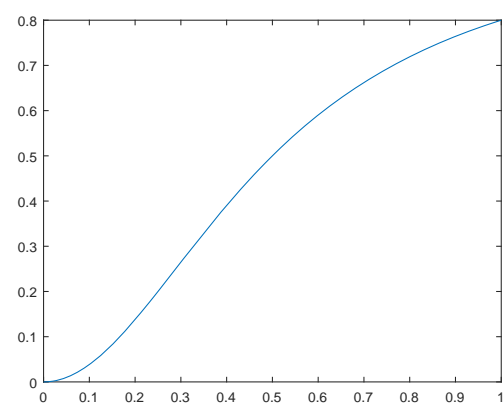

Figure 3. Graph of a Hill function
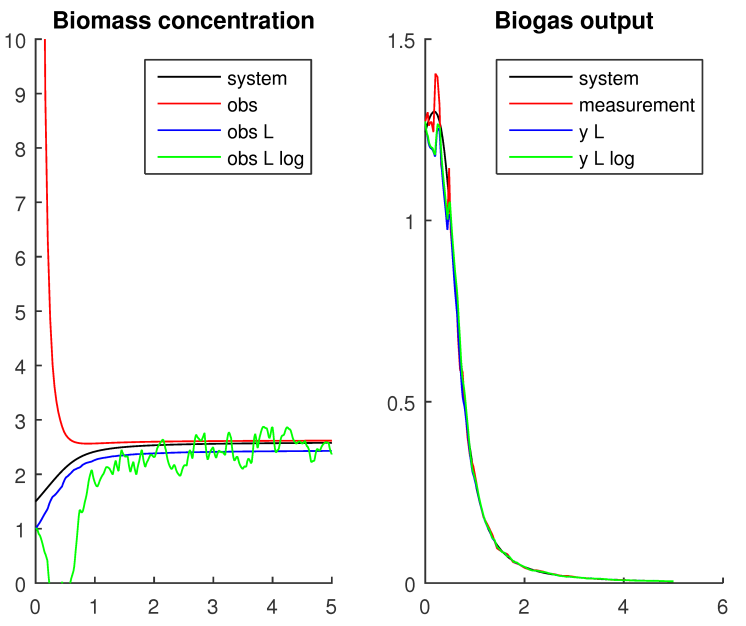

Figure 4. Comparison of the proposed observer with the Luenberger observers (13) and (14) denoted 'L' and 'L log' (with Hill function)

Luenberger observers. However for each positive value $m$ of the function $\mu$, one has $\mu^{-1}(m)=\left\{s_{-}(m), s_{+}(m)\right\}$ with $0<s_{-}(m) \leq \sqrt{K_{s} K_{i}} \leq s_{+}(m)$, and as solutions of system (1) converge to $s=0$, we have considered $\mu^{-1}(m)=s_{-}(m)$ in the Luenberger observers (13) and (14). This gives the right inverse as soon as $t$ satisfies $s(t) \leq \sqrt{K_{s} K_{i}}$ ), but this penalizes the transients as this can be seen on simulations of Fig. 6, that have been ran for $\mu_{0}=1, K_{s}=1$ and $K_{i}=0.2$.

These simulations show that the asymptotic observer works satisfactorily in simulation for a large variety of growth functions, with a good behavior with respect to measurement noise. Let us underline that it does not require a multiplicative gain on the measurement as for the Luenberger observers or any "classical" observers. One can note that for the Haldane function, the property of having an upper estimation of the biomass is no longer satisfied, in accordance with Remark 4.2.

\section{B. Robust observer}

In this section we consider noise with an amplitude five time larger than in the previous simulations. In such cases, the error on the initial measurement $y(0)$ can impact significantly

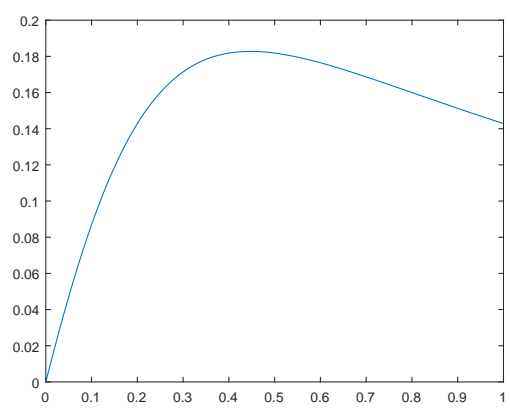

Figure 5. Graph of a Haldane function
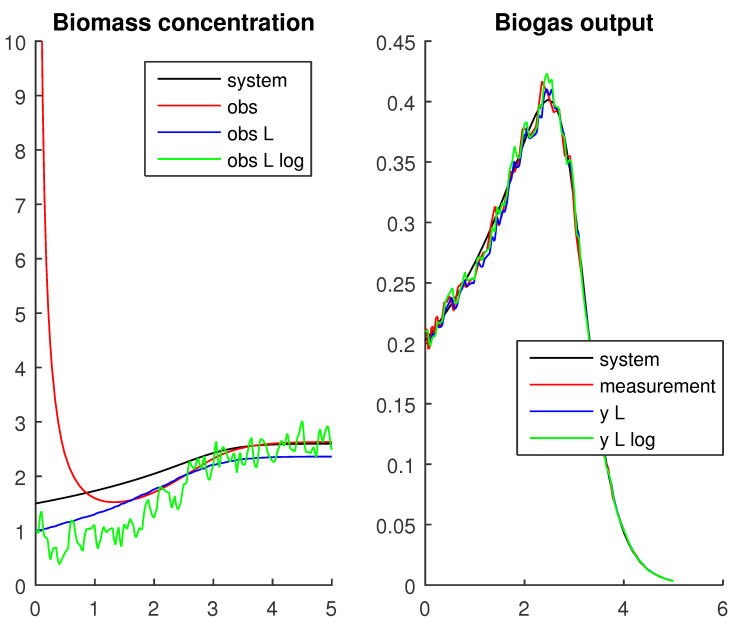

Figure 6. Comparison of the proposed observer with the Luenberger observers (13) and (14) denoted 'L' and 'L log' (with Haldane function)

the asymptotic bias of the asymptotic observer (4.2) (see the expression (9) given in Section V). We have compared the robust version of the asymptotic observer proposed in (11) with $T=0.1$ and $N=10$, for the same growth functions and parameters of the simulations previously presented (see Fig. 7, 8 and 9).

The simulations show that with a relatively small value of $N$, the approximated robust observer can cope quite well with a large deviation of initial measurement and noise.

Note that the proposed observer does not rely on the innovation (i.e. the difference between the observation predicted by the observer and the real observation) as usual observers do. This is both a weakness and an advantage, because the innovation informs about the estimation error in real-time, but on the other hand our observer does not suffer from noise sensitivity and peaking phenomenon. However let us underline that its convergence is governed by the integral term $\int_{t_{0}}^{t} y(\tau) d \tau$ (see expression (6)). Therefore, when this term does change much over time, this means that the estimator has roughly converged. 

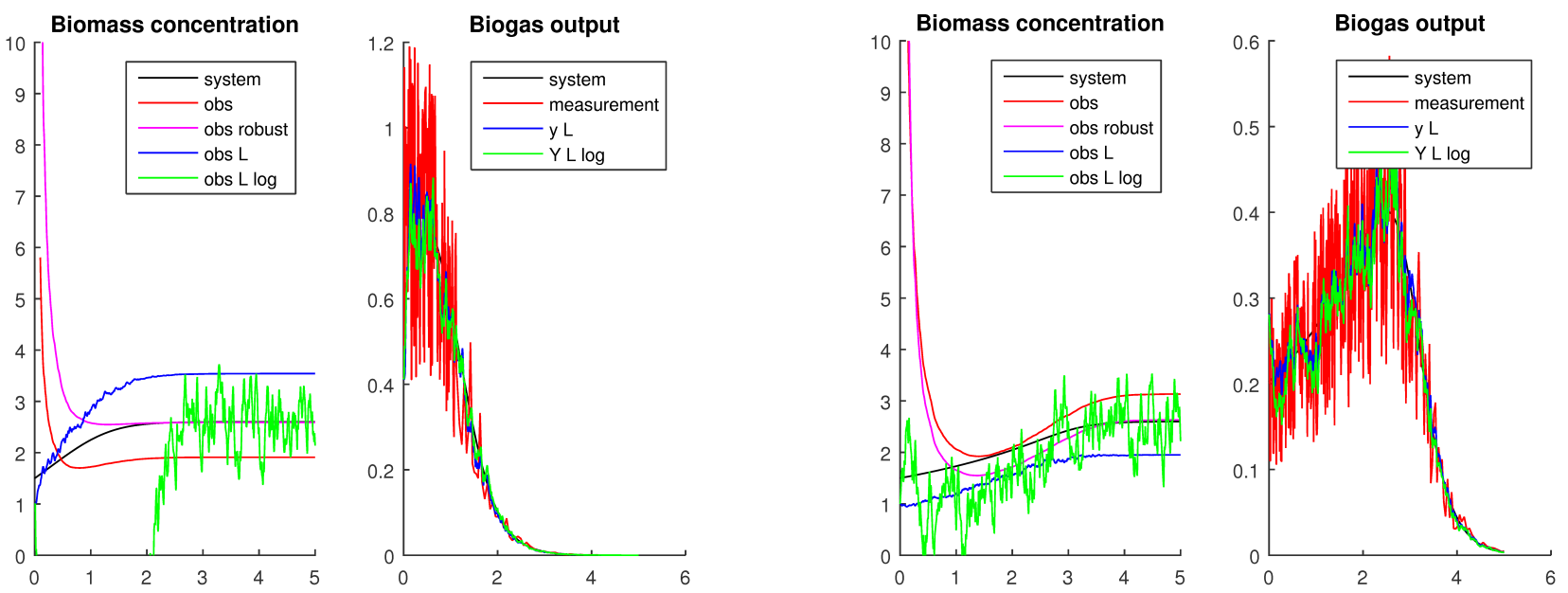

Figure 7. Comparison with the robust asymptotic observer under strong noise (with Monod function)
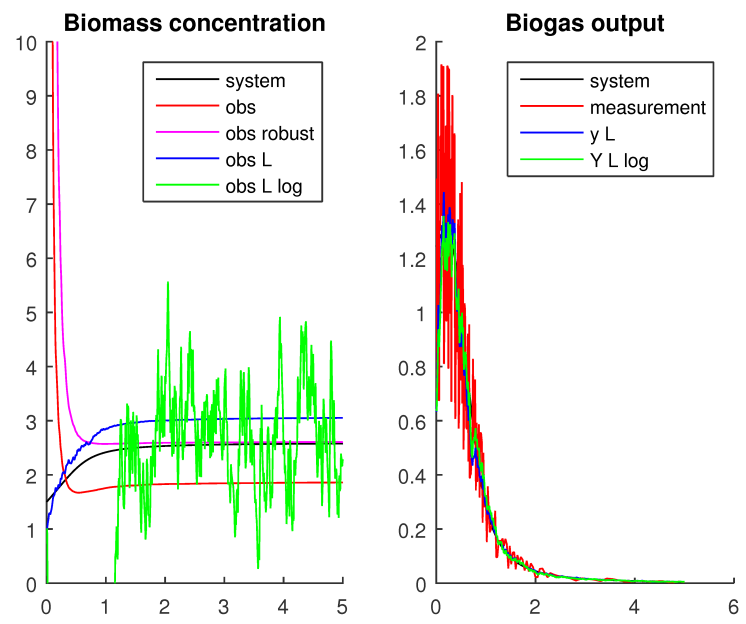

Figure 8. Comparison with the robust asymptotic observer under strong noise (with Hill function)

\section{CONCLUSIONS}

In this work, we have proposed an observer for a system that is not observable on its set of asymptotic states. To cope with this difficulty, our observer is of a particular structure which does not use the innovation as the usual observers do. Its convergence speed cannot be freely addressed but we prove its exact convergence for any initial condition different from an asymptotic state. Moreover we have proposed a robust extension of this observer in higher dimension to deal with measurement disturbances. Numerical simulations have shown the good performances of the proposed observer on a biological system of practical interest, compared to other solutions such as Luenberger observers. Consideration of larger classes of systems with similar lack of observability will be the matter of a future work.

Acknowledgments. The authors would like to thank the $\mathrm{Hu}$ bert Curien exchange program TOURNESOL between France

Figure 9. Comparison with the robust asymptotic observer under strong noise (with Haldane function)

and Belgium for his support. The scientific responsibility rests with its authors.

\section{REFERENCES}

[1] J. Ali, N. Hoang, M. Hussain And D. Dochain, Review and classification of recent observers applied in chemical process systems, Computers \& Chemical Engineering, 76, pp. 27-41, 2015.

[2] G. BAstin AND D. Dochain, On-line Estimation and Adaptive Control of Bioreactors, Elsevier, 1990.

[3] O. Bernard, Z. Hadj-Sadok, D. Dochain, A. Genovesi and J.P. STEYER, Dynamical model development and parameter identification for an anaerobic wastewater treatment process, Biotechnology \& Bioengeeniring, 75(4), pp. 424-438, 2001.

[4] S. Biagiola And J. Solsona, State estimation in batch processes using a nonlinear observer, Mathematical and Computer Modelling, 44(11), pp. 1009-1024, 2006.

[5] J.-P. Coriou, Process Control. Theory and Applications, Springer, 2004.

[6] D. Dochain, State and parameter estimation in chemical and biochemical processes: A tutorial, Journal of Process Control, 13, pp. 801-818, 2003.

[7] D. DOCHAIN AND A. RAPAPORT, An asymptotic observer for batch processes with single biogas measurement, IFAC Conference on Modelling, Identification and Control of Nonlinear Systems (MICNON), Guadalajara, Mexico, 20-22 June 2018.

[8] J.-P GaUthier, H. HAMmouri AND S. OTHMAN, A simple observer for nonlinear systems applications to bioreactors, IEEE Transactions on automatic control, 37 (6), pp. 875-880, 1992.

[9] J.-P. GAUTHIER AND A.K. KUPKA, Observability and observers for nonlinear systems, SIAM J. Control and Optimization, 32(4), pp. 975994, 1998.

[10] G.R. Gavalas, Nonlinear Differential Equations of Chemically Reacting Systems, Springer Verlag, Berlin, 1968.

[11] N. KAZANTZIS AND C. KRAVARIS, Nonlinear observer design using Lyapunov's auxiliary theorem, Systems and Control Letters, 34(5), pp. 241-247, 1998.

[12] A. Holmberg And J. Ranta, Procedures for parameter and state estimation of microbial growth process models, Automatica, 18, pp. 181193, 1982

[13] A. KRENER AND A. ISIDORI, Linearization by output injection and nonlinear observers, Systems and Control Letters, 3(1), pp. 47-52, 1983.

[14] A. KRener, Nonlinear Observers, In Control Systems, Robotics and Automation, edited by H. Unbehauen, in Encyclopedia of Life Support Systems (EOLSS), 21 p., 2004.

[15] D.G. Luenberger, An Introduction to Observers, IEEE Transactions on Automatic Control, 16, pp. 596-602, 1971. 
[16] J. Moreno And D. Dochain, Global observability and detectability analysis of uncertain reaction systems and observer design, International Journal of Control, 81(7), pp. 1062-1070, 2008.

[17] K. PREUSS AND M. LE LANN, Growth rate estimation in batch culture of brevibacterium linens, 8th Int. Conf. on Computer Applications in Biotechnology (CAB'8), Québec (Canada), 24-27 June, pp. 347-352, 2001.

[18] A. RAPAPORT AND M. MALOum, Design of exponential observers for nonlinear systems by embedding, International Journal of Robust and Nonlinear Control, 14, pp. 273-288, 2004.

[19] A. Schaum And J. Moreno, Dynamical analysis of global observability properties for a class of biological reactors, IFAC Proceedings Volumes 40 (4), pp. 213-218, 2008.

[20] A. Schaum, J. Moreno, J. Díaz-Salgado and J. Alvarez, Dissipativity-based observer and feedback control design for a class of chemical reactors, Journal of Process Control 18 (9), pp. 896-905, 2008.

[21] H. Shimizu, T. Takamatsu, S. Shioya and K. Suga, An algorithmic approach to constructing the on-line estimation system for the specific growth rate, Biotechnology and Bioengineering, 33, pp. 354364, 1989.

[22] M. SOROUSH Nonlinear state-observer design with application to reactors, Chemical Engineering Science, 52 (3), pp. 387-404, 1997.

[23] M. VIDYASAGAR, On the stabilization of nonlinear systems using state detection, IEEE Trans. on Aut. Cont., 25(3), pp. 504-509, 1980. 